\title{
Internet non-use among the Canadian older adult population: General Social Survey (GSS)
}

\author{
Hossam Ali-Hassan
}

Vineeth Sekharan

\section{Theresa Kim}

Assistant Professor: Glendon College, York University, Toronto, Canada.

Corresponding Author. hossama@glendon.yorku.ca

Research Associate: York University, Toronto, Canada.

vineeth.sekharan@mail.utoronto.ca

Post-doctoral research fellow: The Hospital for Sick Children, Research Institute, Toronto, Canada

theresa.kim@sickkids.ca

Benefits of Internet use for older adults include the ability to access informational resources, facilitate social connections and use online communication resources. Further research on identifying the characteristics of older adult Internet non-users is warranted. The present study aims to examine the prevalence and characteristics of Internet non-use among Canada's older adult populations. The analysis was based on the 2016 General Social Survey (GSS)Canadians at Work and Home. Analysis was restricted to Canadians of 65 years of age or older. The outcome was Internet non-use, which was defined as having not used the Internet in the 30-day period prior to survey data collection. Demographic, socio-economic, health related, and social support and relationship factors were considered for a multivariable logistic regression analysis.

Ali-Hassan, H., Sekharan. V., Kim. T. (2019). Internet non-use among the Canadian older adult population: General Social Survey (GSS). The Journal of Community Informatics, 15, 61-77.

Date submitted: 2018-03-31. Date accepted: 2018-10-31.

Copyright (C), 2019 (the authors as stated). Licensed under the Creative Commons AttributionNonCommercial-ShareAlike 2.5. Available at: www.ci-journal.net/index.php/ciej/article/view/1447 
Overall, the prevalence of Internet non-use among Canadian older adults was $31.9 \%$. Characteristics significantly associated with Internet non-use included: lower educational achievement, decreased socioeconomic status, poor mental and physical health, having a partner / significant other, and being a cigarette smoker. The province of residence was significantly associated with non-internet use with residents of Quebec being at increased odds of non-internet use compared to residents of British Columbia $(O R=2.09$, 95\% CI= 1.51-2.88). Additionally, increased age among older adults was associated with increased likelihood of not using the Internet. The findings from this study can be used as the basis for future research and to aid in the development of effective policies and programs directed towards the needs of this unique population.

\section{Introduction}

The benefits of Internet use are multifold, and include the ability to access informational resources, facilitate social connections, use banking and retail services, and consume audiovisual media. However, Internet use varies greatly among different demographics and population groups, with lower adoption rates present in older adult Canadian households (Middleton \& Sorenson, 2006). This is despite older adult populations being capable of reaping many of the same benefits from the Internet as their younger counterparts, which include accessing social supports, reducing loneliness, as well as achieving better life satisfaction and psychological well-being (Heo et al., 2015).

As populations age, social isolation has been found to progressively increase (Lelkes, 2012). Older adult use of the Internet has been strongly associated with significant decreases in social isolation levels and reduced risk of depression (Cotton et al., 2014; Tsai et al., 2015; Khosravi, Rezvani \& Wiewiora, 2016; Chopik, 2016). Lelkes (2012) analyzed cross-sectional data from the European Social Survey, and found that Internet use among older adults corresponded with higher scores of life satisfaction, increased happiness and reduced social isolation, even when controlling for potential confounding variables like income and education. The study also found that use of the Internet may help facilitate increased social interactions offline (Lelkes, 2012). These findings suggest that the benefits of Internet use and associated information technologies is associated with positive social effects for older adults.

The Technology Acceptance Model (TAM), proposed by Davis (1989), identifies numerous factors that influence decisions to use technology, with a focus on the perceived usefulness and ease-of-use of a technology. Identified factors of technology acceptance among older adults include usability, gender, perceived benefits of use and socio-demographic markers (Ma et al., 2015; Arenas-Gaitan, Peral-Peral \& Jeronimo, 2015; Niehaves \& Plattfaut, 2014). As noted by McDonough (2016), the presence of disparities in use rates of the Internet among older adults varies by ethnicity, education and income. Similar findings are present throughout published literature in studies 
conducted in the United States and Europe, establishing that older adults are a heterogeneous group, with diverse traits and characteristics, some of which are closely associated with digital exclusion (van Deursen \& Helsper, 2015; Yu et al., 2016; van Boekel, Peek \& Luijkx, 2017).

As the Internet and its use becomes increasingly integrated with everyday life, failure to recognize and address disparities among older adults in computer literacy, perceived usefulness of Internet use, and barriers to Internet access will only broaden existing digital divides that exist within older adult populations (McDonough, 2016). For example, the Internet plays an increasingly important role in the development of new health technologies and informational resources for older adults (Bujnowska-Fedak \& Mastalerz-Migas, 2014). This includes advances in patient monitoring, mobile health applications in healthcare, and online applications designed to promote improved medication adherence and mental health (Ray, 2014; Hussain et al., 2014). Failure to identify and address existing discrepancies will only further disadvantage current subsets of the Canadian older adult population who do not use the Internet.

To date, there has been limited analysis on the prevalence and characteristics of Internet non-use in older adult populations in Canada. By elucidating the factors that impact Internet use in the country, better services and targeted programs that facilitate uptake of new technologies among older adults may be provided. Therefore, the aim of this study is to examine and identify prevalence and characteristics of Internet use among older adults in Canada, using the nationally representative General Social Survey.

\section{Methods}

\section{Study Design and Data Collection}

This study used data from Canada's 2016 General Social Survey (GSS) - Canadians at Work and Home. Administered once every five years, the GSS currently contains focused questions on caregiving, families, time use, social identity, volunteering and victimization as well as a standardized set of socio-demographic questions. The survey is administered by Statistics Canada, a federal government agency commissioned with gathering statistical data and producing statistical reports. The objectives of the GSS are to collect data to monitor changes in social trends, as well as the general well-being of Canadians. Survey data was collected between August 2, 2016 and December 23, 2016.

The GSS has a cross-sectional survey design that used stratified sampling with a twostage sampling design from all the Canadian provinces. At each of the sampling stages, Statistics Canada employed probability sampling with random selection. Units in the first sampling stage consisted of groups of telephone numbers that were assigned to households based on a frame that combined landline and cellular telephone numbers from various administrative sources within Statistics Canada's sampling frame. Units in the second stage of sampling consisted of individuals within each of the identified households. For each household, only one non-institutionalized person of 15 years or 
older, was eligible to complete the GSS. Interviews were primarily conducted in English and French, using a Computer Assisted Telephone Interview (CATI). The design and methods of CATI techniques have been described in further detail elsewhere in the literature (Choi, 2004; St. Pierre \& Beland, 2008; LaFlamme \& Karagannis, 2010).

Ethics approval for the study was not required as analysis was based on a secondary analysis of GSS data collected by Statistics Canada. Access to survey data was obtained through the Research Data Centre in Toronto, Canada.

\section{Measures}

For the present study, data analysis was restricted to older adults defined as a person of 65 years of age and older (Ramage-Morin, 2009; Quaan-Haase, Mo \& Wellman, 2017; Statistics Canada, 2017). The main outcome variable of this study was Internet non-use. Specifically, this was collected by the GSS in a question that asked respondents, "In the past month, how often have you used the internet?" This outcome was dichotomized into: "used Internet" (includes responses of "daily", "a few times a week", "once a week", "few times in a month") and "did not use in the past month." Independent variables included demographic, socioeconomic, health-related, and social support and relationship factors. Demographic factors included age, sex, living arrangement, province of residence, immigrant status, and Aboriginal status. Socioeconomic factors included employment status, educational achievement and social class standing. Healthrelated variables included consumption of alcoholic beverages in the past month, current status of cigarette smoking, general health status, mental health status, and amount of stress. Social support and relationship variables included social support, satisfaction with personal relationships, and marital status. Social support was assessed by asking respondents if they had people in their lives who they felt they could depend on. Marital status was dichotomized into "having a partner/significant other" (includes responses of "single, "never married", "widowed", "separated", and "divorced") and "not having a partner/significant other" (includes responses of "married" and "common law"). Data for both the outcome and the independent variables were collected through self-report.

\section{Statistical Analysis}

Chi square tests were conducted to determine bivariate associations between different levels of each of the demographic, socioeconomic, health-related, and social support and relationships factors and reported Internet non-use among older adults. To assess which factors were independently associated with non-Internet use, a multivariable logistic regression model was conducted with the outcome being non-Internet use and the independent variables being all the demographic, socioeconomic, health-related, and social support and relationships factors. Population weights were applied to all estimates to make the results representative of the target population at the time of the survey. To account for the complex sampling design, bootstrapping was performed to calculate the $95 \%$ CI estimates. Population weights and bootstrap weights were created 
by Statistics Canada and provided with the GSS data file. All analyses were conducted with Stata Data Analysis and Statistical Software (Stata, version 13.0) and set at alpha $<0.05$ for two-tailed tests of statistical significance.

\section{Results}

For the present study the weighted sample size analyzed was 5,782,818 older Canadian adults. Figure 1 shows the distribution of Internet non-use by provincial groupings, as well as the overall average for Internet non-use across all provinces. The overall rate of Internet non-use in Canada, for older adults, was 31.9\%. The rates of Internet non-use ranged between provinces from a low of $23.5 \%$ in British Columbia to a high of $38.5 \%$ in Quebec.

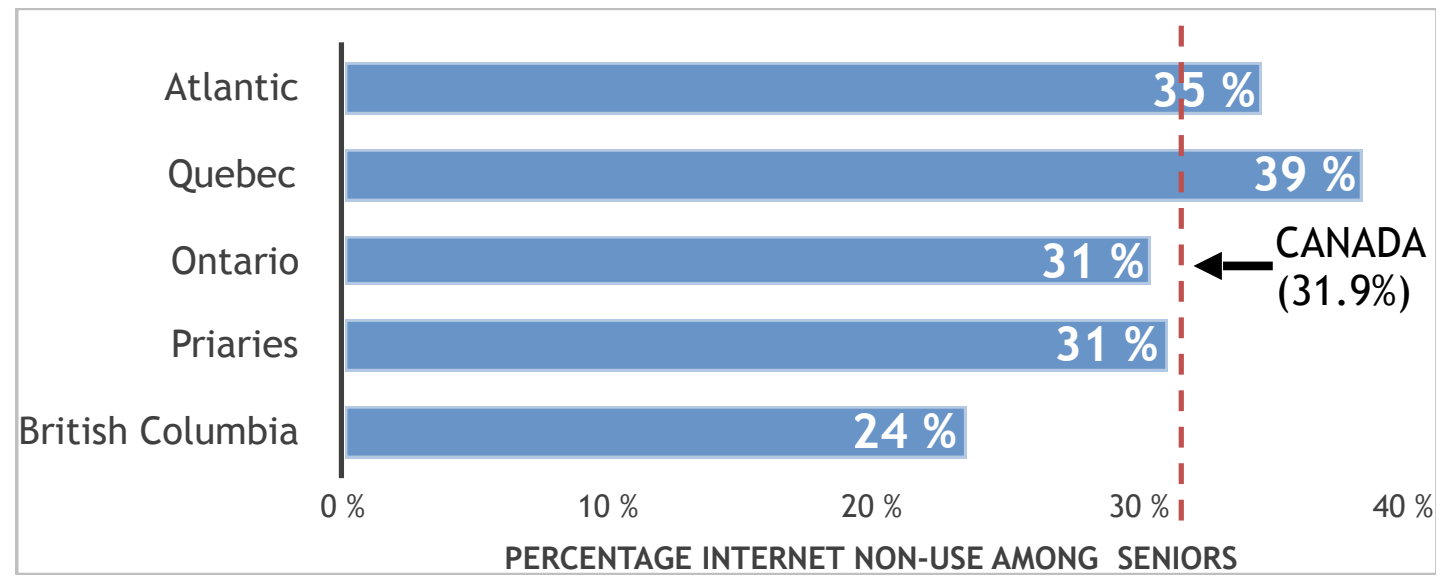

Figure 1: Percentage of Internet Non-use Among Older Adults Across Canadian Provinces

Table 1 presents the descriptive data and unadjusted analysis of potential characteristics, and frequencies associated with Internet non-use among Canadian older adults. Older adults aged 65-69 had the lowest percentage of Internet non-use, at 15.2\%, while the oldest age group, older adults aged $80+$, had the highest percentage of Internet non-use at $59.1 \%(\mathrm{p}<0.05)$. Internet non-use was also more prominent among female older adults $(35.3 \%)$ than male older adults $(28.0 \%)(\mathrm{p}<0.05)$. Internet non-use was also higher among older adults who were Aboriginal persons (42.4\%), compared to those who were not $(31.7 \%)(\mathrm{p}<0.05)$. Older adults whose highest education completed was less than high school had much higher rates of Internet non-use (60.4\%), than those who had completed greater than high School $(16.2 \%)(\mathrm{p}<0.05)$. Higher social class standing also corresponded with decreased rates of Internet non-use among older adults, with rates of $40.7 \%$ Internet non-use among lower/low-middle class households and rates of $16.8 \%$ Internet non-use for upper-middle/upper class households $(\mathrm{p}<0.05)$.

Table 1: Descriptive Data and Unadjusted Analysis of Potential Characteristics and Internet Non-use

\begin{tabular}{|c|c|c|c|}
\hline \multirow{2}{*}{} & Total & \multicolumn{2}{|c|}{ Internet Non-use } \\
\cline { 2 - 4 } & $\mathbf{N}(\%)^{\mathrm{a}}$ & $\mathbf{N}(\%)^{\mathrm{a}}$ & P-Value $^{*}$ \\
\hline
\end{tabular}

Demographic Factors 


\begin{tabular}{|c|c|c|c|}
\hline & \multirow{2}{*}{$\begin{array}{c}\text { Total } \\
\mathbf{N}(\%)^{\mathrm{a}}\end{array}$} & \multicolumn{2}{|c|}{ Internet Non-use } \\
\hline & & $\mathbf{N}(\%)^{a}$ & P-Value* \\
\hline $\begin{array}{l}\text { Age (years) } \\
\begin{array}{r}65-69 \\
70-74 \\
75-79 \\
80+\end{array}\end{array}$ & $\begin{array}{l}1,966,315(33.9) \\
1,448,839(25.0) \\
1,020,344(17.6) \\
1,366,338(23.5)\end{array}$ & $\begin{array}{l}297,962(15.2) \\
360,320(24.9) \\
387,353(38.1) \\
799,377(59.1)\end{array}$ & $<0.0001$ \\
\hline $\begin{array}{l}\text { Male } \\
\text { Female }\end{array}$ & $\begin{array}{l}2,678,908(46.2) \\
3,122,928(53.8)\end{array}$ & $\begin{array}{l}747,269(28.0) \\
1,097,742(35.3)\end{array}$ & $<0.0001$ \\
\hline $\begin{array}{l}\text { Living Arrangement } \\
\text { Not living alone } \\
\text { Living alone }\end{array}$ & $\begin{array}{l}4,170,042(71.9) \\
1,631,794(28.1)\end{array}$ & $\begin{array}{l}1,122,400(27.0) \\
722,611(44.5)\end{array}$ & $<0.0001$ \\
\hline $\begin{array}{l}\text { Province } \\
\qquad \begin{array}{l}\text { British Columbia } \\
\text { Prairies }^{\mathrm{b}} \\
\text { Ontario } \\
\text { Quebec } \\
\text { Atlanticc }\end{array}\end{array}$ & $\begin{array}{l}833,037(14.4) \\
840,324(14.5) \\
2,224,345(38.3) \\
1,457,991(25.1) \\
446,139(7.7)\end{array}$ & $\begin{array}{l}195,539(23.5) \\
260,364(31.1) \\
674,324(30.5) \\
560,501(38.5) \\
154,284(34.7)\end{array}$ & $<0.0001$ \\
\hline $\begin{array}{l}\text { Ever Landed Immigrant } \\
\qquad \begin{array}{c}\text { No } \\
\text { Yes }\end{array}\end{array}$ & $\begin{array}{l}4,316,230(74.4) \\
1,485,607(25.6)\end{array}$ & $\begin{array}{l}1,406,769(32.7) \\
438,242(29.6)\end{array}$ & 0.1416 \\
\hline $\begin{array}{c}\text { Aboriginal Person } \\
\qquad \begin{array}{c}\text { No } \\
\text { Yes }\end{array}\end{array}$ & $\begin{array}{l}5,689,279(98.4) \\
94,624(1.6)\end{array}$ & $\begin{array}{l}1,796,035(31.7) \\
39,112(42.4)\end{array}$ & 0.00704 \\
\hline \multicolumn{4}{|l|}{ Socioeconomic Factors } \\
\hline $\begin{array}{c}\text { Currently Employed } \\
\qquad \begin{array}{c}\text { No } \\
\text { Yes }\end{array}\end{array}$ & $\begin{array}{l}5,166,867(89.1) \\
634,969(10.9)\end{array}$ & $\begin{array}{l}1,756,599(34.1) \\
88,412(13.9)\end{array}$ & $<0.0001$ \\
\hline
\end{tabular}




\begin{tabular}{|c|c|c|c|}
\hline & \multirow{2}{*}{$\begin{array}{c}\text { Total } \\
\mathbf{N}(\%)^{\mathrm{a}}\end{array}$} & \multicolumn{2}{|c|}{ Internet Non-use } \\
\hline & & $\mathbf{N}(\%)^{\mathrm{a}}$ & P-Value* \\
\hline $\begin{array}{l}\text { Highest Education Completed } \\
\text { Less than High School } \\
\text { High School } \\
\text { Greater than High Schoold }\end{array}$ & $\begin{array}{l}1,562,873(27.1) \\
1,442,352(25.0) \\
2,756,249(47.9)\end{array}$ & $\begin{array}{l}939,015(60.4) \\
440,705(30.6) \\
445,812(16.2)\end{array}$ & $<0.0001$ \\
\hline $\begin{array}{l}\text { Social Class } \\
\qquad \begin{array}{l}\text { Lower/Lower-Middle } \\
\text { Middle } \\
\text { Upper-Middle/Upper }\end{array}\end{array}$ & $\begin{array}{l}827,393(14.6) \\
3,888,853(68.7) \\
944,095(16.7)\end{array}$ & $\begin{array}{l}334,171(40.7) \\
1,298,141(33.5) \\
158,379(16.8)\end{array}$ & $<0.0001$ \\
\hline Health-Related Factors & & & \\
\hline $\begin{array}{l}\text { Alcohol in Past Month } \\
\qquad \begin{array}{l}\text { None } \\
\text { Once a week/once or twice in } \\
\text { past month } \\
\text { 2-3 times/week or more }\end{array}\end{array}$ & $\begin{array}{l}2,212,085(38.2) \\
1,819,922(31.4) \\
1,756,898(30.4)\end{array}$ & $\begin{array}{l}987,570(44.8) \\
474,993(26.2) \\
375,091(21.4)\end{array}$ & $<0.0001$ \\
\hline $\begin{array}{c}\text { Current Smoking } \\
\qquad \begin{array}{c}\text { No } \\
\text { Yes }\end{array}\end{array}$ & $\begin{array}{l}5,307,681(91.5) \\
489,607(8.5)\end{array}$ & $\begin{array}{l}1,663,015(31.4) \\
181,214(37.2)\end{array}$ & 0.0366 \\
\hline $\begin{array}{l}\text { General Health } \\
\qquad \begin{array}{l}\text { Poor/Fair } \\
\text { Good } \\
\text { Very good/Excellent }\end{array}\end{array}$ & $\begin{array}{l}1,175,267(20.3) \\
2,017,970(24.9) \\
2,589,582(44.8)\end{array}$ & $\begin{array}{l}518,105(44.4) \\
697,067(34.6) \\
624,678(24.2)\end{array}$ & $<0.0001$ \\
\hline $\begin{array}{l}\text { Mental Health } \\
\qquad \begin{array}{l}\text { Poor/Fair } \\
\text { Good } \\
\text { Very good/Excellent }\end{array}\end{array}$ & $\begin{array}{l}358,091(6.2) \\
1,895,490(32.8) \\
3,527,193(61.0)\end{array}$ & $\begin{array}{l}188,197(52.6) \\
756,031(40.1) \\
888,811(25.3)\end{array}$ & $<0.0001$ \\
\hline $\begin{array}{c}\text { Amount of Stress on Most Days } \\
\text { Not Stressful } \\
\text { Stressful }\end{array}$ & $\begin{array}{l}3,534,157(61.4) \\
2,224,111(38.6)\end{array}$ & $\begin{array}{l}1,110,710(31.6) \\
717,279(32.3)\end{array}$ & 0.6496 \\
\hline
\end{tabular}




\begin{tabular}{|c|c|c|c|}
\hline & \multirow{2}{*}{$\begin{array}{c}\text { Total } \\
\mathbf{N}(\%)^{\mathrm{a}}\end{array}$} & \multicolumn{2}{|c|}{ Internet Non-use } \\
\hline & & $N(\%)^{a}$ & P-Value* \\
\hline $\begin{array}{l}\text { People You Can Depend On } \\
\text { Never/Rarely/Sometimes } \\
\text { Often } \\
\text { Always }\end{array}$ & $\begin{array}{l}1,011,725(17.6) \\
1,498,622(26.1) \\
3,239,925(56.3)\end{array}$ & $\begin{array}{l}360,233(35.7) \\
397,146(26.6) \\
1,063,105(32.9)\end{array}$ & 0.0004 \\
\hline $\begin{array}{l}\text { Satisfaction with Personal } \\
\text { Relationships } \\
\text { Not really satisfied } \\
\text { Moderately satisfied } \\
\text { Completely satisfied }\end{array}$ & $\begin{array}{l}2,862,375(49.8) \\
975,340(17.0) \\
1,912,691(33.2)\end{array}$ & $\begin{array}{l}871,767(30.5) \\
253,390(26.0) \\
690,801(36.3)\end{array}$ & $<0.0001$ \\
\hline $\begin{array}{c}\text { Marital Status } \\
\qquad \text { No } \\
\text { Yes }\end{array}$ & $\begin{array}{l}1,957,575(33.7) \\
3,844,261(66.3)\end{array}$ & $\begin{array}{l}865,593(44.5) \\
979,418(25.5)\end{array}$ & $<0.0001$ \\
\hline
\end{tabular}

a Sample sizes are estimated using normalized weights, ${ }^{b}$ Prairies provinces include Alberta, Manitoba, and Saskatchewan, ${ }^{c}$ Atlantic provinces include New Brunswick, Nova Scotia, Newfoundland, and Prince Edward Island, d Greater than High School indicates those with Trade Certificate, College, University, or Bachelor and above, *Bolded values and CIs denote significance $(\mathrm{p}<0.05)$

Table 2 shows the unadjusted and adjusted Odds Ratios (ORs) of Internet non-use among older adults in Canada. In the adjusted model, when compared to the youngest age sub-group among older adults (65-70), increased age was associated with increased odds of not using the Internet for older adults aged 70-74 (OR: 1.83, 95\% CI: 1.41-2.37), for older adults aged 75-79 (OR: 3.42, 95\% CI: 2.61-4.48) and for older adults aged 80 years or older (OR: 6.74, 95\% CI: 5.08-8.95). When compared to residing in the province of British Columbia, those residing in the province of Quebec were at increased odds of Internet non-use, after adjustment to all other factors (OR: 2.09, 95\% CI: 1.51-2.88). Reduced educational achievement was associated with increased odds of Internet non-use for older adults whose highest education completed was high school (OR: 1.95, 95\% CI: 1.56-2.43) and less than high School (OR: 5.05, 95\% CI: 4.01-6.36) when compared to older adults whose educational achievement was greater than high School.

Lower social class standing was also associated with increased odds of Internet non-use when compared to upper-middle and upper class status, for both older adults who were middle class (OR: 1.82, 95\% CI: 1.36-2.44) and lower/lower-middle class (OR: 2.01, 95\% CI: 1.38-2.94). In the adjusted model, alcohol use was associated with reduced Internet non-use whereas current smoking was associated with increased odds of Internet non-use. Poor/fair general health and mental health were associated with increased Internet non-use was associated with poor/fair general health (OR: 1.46, 95\% CI: 1.11-1.91 poor/fair general health compared to very/good/excellent health) and (OR: 
2.19, 95\% CI: $1.44-3.32$ for poor/fair mental health very good/excellent mental health). When analyzing the relationship between satisfaction with personal relationships and Internet non-use, being completely satisfied with relationships was associated with Internet non-use (1.30, 95\% CI: 1.05-1.62). After adjusting for other independent variables, not having a partner or significant other was also associated with increased likelihood of Internet non-use (OR: 1.49, 95\% CI: 1.01-2.19).

Table 2: Unadjusted and adjusted Odds Ratio of Potential Characteristics and Internet Non-use among Older Adults in Canada ( $\left.=5,498,203^{\mathrm{a}}\right)$

\begin{tabular}{|c|c|c|}
\hline & $\begin{array}{c}\text { Unadjusted Odds } \\
\text { Ratio }\end{array}$ & $\begin{array}{c}\text { Adjusted Odds } \\
\text { Ratio }\end{array}$ \\
\hline & OR $(95 \% C I)^{*}$ & OR $(95 \% \mathrm{CI})^{*}$ \\
\hline \multicolumn{3}{|l|}{ Demographic Factors } \\
\hline $\begin{array}{l}\text { Age (years) } \\
\qquad \begin{array}{c}65-69 \text { (Ref) } \\
70-74 \\
75-79 \\
80+\end{array}\end{array}$ & $\begin{array}{l}1 \\
1.85(1.48,2.32) \\
3.44(2.72,4.33) \\
8.07(6.54,10.11)\end{array}$ & $\begin{array}{l}1 \\
1.83(1.41,2.37) \\
3.42(2.61,4.48) \\
6.74(5.08,8.95)\end{array}$ \\
\hline $\begin{array}{l}\text { Male (Ref) } \\
\text { Female }\end{array}$ & $\begin{array}{l}1 \\
1.40(1.20,1.63)\end{array}$ & $\begin{array}{l}1 \\
1.13(0.93,1.38)\end{array}$ \\
\hline $\begin{array}{l}\text { Living Arrangement } \\
\text { Not living alone (Ref) } \\
\text { Living alone }\end{array}$ & $\begin{array}{l}1 \\
2.17(1.88,2.50)\end{array}$ & $\begin{array}{l}1 \\
1.09(0.75,1.58)\end{array}$ \\
\hline $\begin{array}{l}\text { Province } \\
\qquad \begin{array}{l}\text { British Columbia (Ref) } \\
\text { Prairies }^{\mathrm{b}} \\
\text { Ontario } \\
\text { Quebec } \\
\text { Atlanticc }\end{array}\end{array}$ & $\begin{array}{l}1 \\
1.46(1.13,1.90) \\
1.42(1.11,1.82) \\
2.04(1.58,2.63) \\
1.72(1.34,2.21)\end{array}$ & $\begin{array}{l}1 \\
1.35(0.97,1.87) \\
1.33(0.98,1.79) \\
\mathbf{2 . 0 9}(\mathbf{1 . 5 1}, \mathbf{2 . 8 8}) \\
1.33(0.97,1.82)\end{array}$ \\
\hline $\begin{array}{l}\text { Ever Landed Immigrant } \\
\qquad \begin{array}{c}\text { No (Ref) } \\
\text { Yes }\end{array}\end{array}$ & $\begin{array}{l}1 \\
0.87(0.72,1.05)\end{array}$ & $\begin{array}{l}1 \\
1.00(0.79,1.27)\end{array}$ \\
\hline $\begin{array}{l}\text { Aboriginal Person } \\
\qquad \begin{array}{c}\text { No (Ref) } \\
\text { Yes }\end{array}\end{array}$ & $\begin{array}{l}1 \\
1.59(0.95,2.66)\end{array}$ & $\begin{array}{l}1 \\
1.12(0.55,2.28)\end{array}$ \\
\hline
\end{tabular}




\begin{tabular}{|c|c|c|}
\hline & $\begin{array}{c}\text { Unadjusted Odds } \\
\text { Ratio }\end{array}$ & $\begin{array}{c}\text { Adjusted Odds } \\
\text { Ratio }\end{array}$ \\
\hline & OR $(95 \% \mathrm{CI})^{*}$ & OR $(95 \% \mathrm{CI})^{*}$ \\
\hline \multicolumn{3}{|l|}{ Socioeconomic Factors } \\
\hline $\begin{array}{c}\text { Currently Employed } \\
\text { No (Ref) } \\
\text { Yes }\end{array}$ & $\begin{array}{l}1 \\
0.31(0.23,0.42)\end{array}$ & $\begin{array}{l}1 \\
0.82(0.58,1.16)\end{array}$ \\
\hline $\begin{array}{l}\text { Highest Education Completed } \\
\text { Less than High School } \\
\text { High School } \\
\text { Greater than High School (Ref)d }\end{array}$ & $\begin{array}{l}7.90(6.52,9.57) \\
2.28(1.88,2.78) \\
1\end{array}$ & $\begin{array}{l}5.05(4.01,6.36) \\
1.95(1.56,2.43) \\
1\end{array}$ \\
\hline $\begin{array}{l}\text { Social Class } \\
\qquad \begin{array}{l}\text { Lower/Lower-Middle } \\
\text { Middle } \\
\text { Upper-Middle/Upper (Ref) }\end{array}\end{array}$ & $\begin{array}{l}3.40(2.56,4.52) \\
2.49(1.98,3.13) \\
1\end{array}$ & $\begin{array}{l}2.01(1.38,2.94) \\
1.82(1.36,2.44) \\
1\end{array}$ \\
\hline \multicolumn{3}{|l|}{ Health-Related Factors } \\
\hline $\begin{array}{l}\text { Alcohol in Past Month } \\
\qquad \begin{array}{l}\text { None (Ref) } \\
\text { Once a week/once or twice in past } \\
\text { month } \\
\text { 2-3 times/week or more }\end{array}\end{array}$ & $\begin{array}{l}1 \\
0.44(0.36,0.53) \\
0.33(0.28,0.41)\end{array}$ & $\begin{array}{l}1 \\
0.57(0.46,0.71) \\
0.61(0.48,0.77)\end{array}$ \\
\hline $\begin{array}{c}\text { Current Smoking } \\
\text { No (Ref) } \\
\text { Yes }\end{array}$ & $\begin{array}{l}1 \\
1.29(1.03,1.62)\end{array}$ & $\begin{array}{l}1 \\
1.90(1.39,2.59)\end{array}$ \\
\hline $\begin{array}{l}\text { General Health } \\
\qquad \begin{array}{l}\text { Poor/Fair } \\
\text { Good } \\
\text { Very good/Excellent (Ref) }\end{array}\end{array}$ & $\begin{array}{l}2.51(2.06,3.04) \\
1.66(1.39,1.98) \\
1\end{array}$ & $\begin{array}{l}\mathbf{1 . 4 6}(\mathbf{1 . 1 1}, \mathbf{1 . 9 1}) \\
1.24(0.98,1.57) \\
1\end{array}$ \\
\hline $\begin{array}{l}\text { Mental Health } \\
\qquad \begin{array}{l}\text { Poor/Fair } \\
\text { Good } \\
\text { Very good/Excellent (Ref) }\end{array}\end{array}$ & $\begin{array}{l}3.28(2.46,4.38) \\
1.98(1.68,2.34) \\
1\end{array}$ & $\begin{array}{l}2.19(1.44,3.32) \\
1.38(1.10,1.72) \\
1\end{array}$ \\
\hline $\begin{array}{c}\text { Amount of Stress on Most Days } \\
\qquad \begin{array}{c}\text { Not Stressful (Ref) } \\
\text { Stressful }\end{array}\end{array}$ & $\begin{array}{l}1 \\
1.04(0.88,1.21)\end{array}$ & $\begin{array}{l}1 \\
0.91(0.75,1.11)\end{array}$ \\
\hline
\end{tabular}




\begin{tabular}{|c|c|c|}
\hline & $\begin{array}{c}\text { Unadjusted Odds } \\
\text { Ratio }\end{array}$ & $\begin{array}{c}\text { Adjusted Odds } \\
\text { Ratio }\end{array}$ \\
\hline & OR $(95 \% \mathrm{CI})^{*}$ & OR $(95 \% \mathrm{CI})^{*}$ \\
\hline \multicolumn{3}{|l|}{ Social Support and Relationship Factors } \\
\hline $\begin{array}{l}\text { People You Can Depend On } \\
\text { Never/Rarely/Sometimes (Ref) } \\
\text { Often } \\
\text { Always }\end{array}$ & $\begin{array}{l}1 \\
\mathbf{0 . 6 5}(\mathbf{0 . 5 1}, \mathbf{0 . 8 2}) \\
0.88(0.72,1.09)\end{array}$ & $\begin{array}{l}1 \\
0.85(0.64,1.14) \\
1.02(0.78,1.32)\end{array}$ \\
\hline $\begin{array}{l}\text { Satisfaction with Personal Relationships } \\
\text { Not really satisfied (Ref) } \\
\text { Moderately satisfied } \\
\text { Completely satisfied }\end{array}$ & $\begin{array}{l}1 \\
0.80(0.64,1.00) \\
1.30(\mathbf{1 . 0 9}, \mathbf{1 . 5 3})\end{array}$ & $\begin{array}{l}1 \\
0.96(0.73,1.26) \\
\mathbf{1 . 3 0}(\mathbf{1 . 0 5}, \mathbf{1 . 6 2})\end{array}$ \\
\hline $\begin{array}{l}\text { Partner/Significant Other } \\
\qquad \begin{array}{c}\text { No } \\
\text { Yes (Ref) }\end{array}\end{array}$ & $\begin{array}{l}2.34(2.01,2.71) \\
1\end{array}$ & $\begin{array}{l}1.49(1.01,2.19) \\
1\end{array}$ \\
\hline
\end{tabular}

a Sample sizes are estimated using normalized weights, ${ }^{b}$ Prairies provinces include Alberta, Manitoba, and Saskatchewan, ${ }^{\mathrm{c}}$ Atlantic provinces include New Brunswick, Nova Scotia, Newfoundland, and Prince Edward Island, *Bolded values and CIs denote significance $(\mathrm{p}<0.05)$

\section{Discussion}

Overall, $31.9 \%$ of older adults did not use the Internet in the last month at the time of survey. The results document that in Canada, older adults who use the Internet are markedly different from older adults who do not use the Internet. After adjustment, factors identified as being associated with Internet non-use among older adults were: increased age, living in Quebec, lower rates of education, decreased social class standing, decreased alcohol consumption, being a cigarette smoker, poor general health, poor mental health, being completely satisfied with personal relationships, and having single or never married marital status (in comparison to married and divorced marital status). These findings are significant, as older adults who are representative of many of these traits are much less likely to use the Internet than their counterparts, thereby limiting their ability to reap many of the benefits associated with its use.

\section{Demographic Factors}

In the present study, the percentage of Internet non-users increased with age among older adults. While $16 \%$ of older adults aged 65-69 did not use the internet in the previous month, this percentage increased to $25 \%$ among older adults aged $70-74$, and $50 \%$ among older adults aged 75 years of age or older.

This is in line with findings of research studies completed in other developed countries. Research conducted by the Pew Research Center found that after age 75, internet use dropped off significantly, with only $34 \%$ of older adults over 75 using the Internet 
(Zickuhr \& Madden, 2012). Van Deursen and Helsper (2015) conducted a national telephone survey in the Netherlands using random digit dialing and found very similar increases in non-use with age, as their study found non-use in $24.4 \%$ of older adults aged 71-75 and 48\% non-use in older adults over the age of 75 . These findings suggest common trends exist globally among developed countries in Internet non-use among older adults.

\section{Socioeconomic Factors}

The present study found lower socioeconomic status to correspond with lack of Internet use. There are many ways in which low socioeconomic may be related to reduced access and use of the Internet, including: inability to afford Internet-capable devices, difficulty affording the cost of WiFi and data connections, as well as inadequate knowledge and awareness on the potential benefits of Internet use.

This is in line with the findings of studies that have previously investigated the relationship between Internet use by older adult populations and socioeconomic status in other countries (Carpenter \& Buday, 2007; Elliot et al., 2013; Berner et al., 2014; Hargittai, Piper \& Morris, 2018). Carpenter and Buday (2007) examined barriers to Internet use in an older adult US-based retirement community and found that cost can act as a direct barrier to the purchase of Internet-enabled devices, especially for lower income adults. Elliot et al. (2013) conducted a large cross-sectional data using survey data in the United States and found significant differences across socioeconomic groups in the rates of older adults' use of information and communications technology. The digital divide that exists between socioeconomic groups among older adults extends to the general population, both abroad and in Canada. In Canada, 95\% of individuals belonging to the highest income quartile are connected to the Internet, while only $62 \%$ of Canadians in the lowest income quartile have Internet access (CIRA Factbook, 2014).

Additionally, the present study found that lower educational attainment was found to be a factor that corresponded with reduced Internet use among older adults. Similar findings have been found in other studies, albeit not in a Canadian context (Helsper \& Reisdor, 2013; Morris, Goodman \& Brading, 2007; Jensen et al., 2010). Jensen et al. (2010) conducted a study in the midwestern United States that examined whether lowincome adults' utilization of Internet technology was predicted or mediated by numerical and written literacy skills. They found that individuals with lower health literacy skills were less likely to use Internet technology.

\section{Health-Related Factors}

The present study also found cigarette use and none drinking alcohol to correspond with increased odds of not using the Internet. There is no previous literature that discusses the potential relationships that may exist between both of these variables. However, further investigation is warranted, as both cigarette use and alcohol consumption are highly studied behavioural substances, whose use is closely linked to mental and physical well-being outcomes.

In the current study, poorer self-reported overall health among older adults was associated with being less likely to use the Internet. One potential explanation for this finding is that older adults who suffer from debilitating health issues, and disability, are less likely to use the Internet because of difficulties associated with living with their 
physical condition. As noted by Choi and DiNitto (2013), "medical conditions, disabilities, and associated pain that restrict use" are all examples of common physical health related factors that can directly serve as a barrier to Internet use for older adults. Physical symptoms associated with common diseases and increased frailty can directly inhibit the ability of older adults to use the Internet (Mazur et al., 2018). Keränen et al. (2017) conducted a population-based survey to identify how use of information and communication technologies among older adults living with and without frailty differs in Northern Finland. The researchers found that physical frailty was associated with decreased information communication technology use by older adults, independent of educational achievement and opinions about the Internet (Keränen et al., 2017).

Higher scores on mental health items in the GSS survey in the present study were also associated with increased likelihood of using the Internet for older adults. Jung et al. (2010) conducted an investigation that looked at the potential role that psychological factors, such as levels of anxiety and low feelings of self-efficacy, serve as barriers to bridging the digital divide for low-income minority older adults. Jung et al. found that psychological variables, like higher levels of anxiety, served as stronger predictors of enrollment into educational programming and training, than the age of older adults, or even their experience using computers (Jung et al., 2010). Forsman and Nordmyr (2015) conducted a systematic review of conducted qualitative and quantitative research studies that investigated associations between Internet use and mental health among older adults. The synthesis approach adopted by the researchers indicate Internet use is associated with higher scores for self-reported mental health, and associated psychosocial variables like self-efficacy.

\section{Social Support and Relationship Factors}

Interestingly, the present study found that being 'completely satisfied' with personal relationships was significantly associated with increased likelihood of Internet non-use among older adults. While similar findings are not replicated in literature focused on older adults, there are examples in the literature which demonstrate similar themes in the association between relational support and Internet use and access in the general population. For example, a study by Liang, Peng and Yu (2012) studied the relationship that exists between the Internet and overall quality of life in Taiwan for individuals aged 15 and older. One of the key findings from their study was that individuals who regularly accessed and used the Internet had lower satisfaction in the dimension of community support, as it related to quality of life.

In the present study, individuals who were married or in a common-law relationship had increased odds of using the internet when compared to individuals who were single, never married, widowed, separated or divorced. Choi and DiNitto (2013) conducted a similar investigation using a large sample $(\mathrm{N}=6680)$ of US Medicare beneficiaries aged 65 and older, and found that married couples were overrepresented among Internet users. While the reasons for this relationship are unclear, the presence of similar findings elsewhere in the literature, in a different population group, suggest the need for further research to explore the relationship between marital status and Internet use among older adults. 


\section{Strengths and Limitations}

The study had several strengths. First, the data has a representative scope, allowing for generalizability of results across Canadian provinces. Additionally, the relatively large sample size for the GSS allowed ample statistical power to draw meaningful conclusions from the data. The study also controlled for various potential confounding variables in the analysis, including several variables that have not been examined previously in association with Internet use by older adult Canadians. However, there are also major limitations. Firstly, one potential source of information bias is the reliance on self-reporting in the GSS, especially considering that older adult participants in the GSS may be unaware of what activities may constitute Internet use, resulting in potential misclassification bias. Furthermore, since the study was cross-sectional in its nature, causal relationships cannot be inferred based on the significance of study findings.

Lastly, the GSS does not sample Canada's territories which include Nunavut, the Yukon, and the Northwest Territories. Unlike the Canadian provinces, Canadian territories do not have inherent sovereignty, and have their power delegated to them by the federal government. Territories are also more sparsely populated than provinces, and have a significantly greater proportion of individuals who identify themselves as being Aboriginal. While only $4.3 \%$ of the total population of Canada self-identify as being Aboriginal, $64.7 \%$ of residents in the territories self-identify as being Aboriginal (Statistics Canada, 2011). Accordingly, findings are only applicable to older adults living in Canadian provinces and is not representative of residents living in Canadian territories.

\section{Conclusion}

The present study suggests significant differences may exist between Canadian older adults who are Internet users versus non-users. These differences may contribute to adverse living situations, and other social disadvantages, as Internet non-use has been associated with increased social isolation, and reduced access to informational resources like health education resources. Provided the many documented benefits of Internet use for older adults, emphasis needs to be placed on addressing existing disparities.

One of the more interesting findings from the study was that individuals who were married or in a common-law relationship had increased odds of using the internet when compared to individuals who were single, never married, widowed, separated or divorced. A potential reason for this finding may be that older adults in a married or common-law relationship are more likely to use the Internet because they have the support of their partner to help address any technical issues they may face. The use of qualitative methods in a future study would allow researchers to directly ask older adult couples who are married or in a common-law relationship about what role, if any, they feel their partner has on their Internet use, and general acceptance towards the use of information communication technologies. The Technology Acceptance Framework (TAM), widely used in the literature, can be used in such a study to better understand how perceived usefulness, ease of use, and intention to use may contribute to actual 
Internet use or non-use among older adults who are single when compared to older adults in a relationship.

More in depth qualitative and quantitative studies should aim to understand common traits possessed by older adults who do not use the Internet, as well as their individual needs, to inform and direct services and policies. Conducting a detailed needs-based analysis of older adults who are Internet non-users can identify existing barriers to Internet use. It is recommended that the design of such a study should be mindful of the everyday realities of older adults who do not use the internet, and provide an opportunity for respondents to detail the need for supports (ex: technical assistance, peer encouragement, educational supports).

\section{References}

Arenas-Gaitán, J., Peral-Peral, B., \& Jerónimo, M. (2015). Elderly and internet banking: An application of UTAUT2. Journal of Internet Banking and Commerce, 20(1), 1-23.

Berner, J., Rennemark, M., Jogréus, C., Anderberg, P., Sköldunger, A., Wahlberg, M., \& Berglund, J. (2015). Factors influencing Internet usage in older adults (65 years and above) living in rural and urban Sweden. Health informatics journal, 21(3), 237-249.

Bujnowska-Fedak, M. M., \& Mastalerz-Migas, A. (2014). Usage of medical internet and ehealth services by the elderly. In Environment Exposure to Pollutants (pp. 75-80). Springer, Cham.

Carpenter, B. D., \& Buday, S. (2007). Computer use among older adults in a naturally occurring retirement community. Computers in Human Behavior, 23(6), 3012-3024.

Choi, B. C. (2004). Computer assisted telephone interviewing (CATI) for health surveys in public health surveillance: methodological issues and challenges ahead. Chronic Diseases and Injuries in Canada, 25(2), 21.

Choi, N. G., \& Dinitto, D. M. (2013). Internet use among older adults: association with health needs, psychological capital, and social capital. Journal of medical Internet research, 15(5).

CIRA Factbook. (2014). The Canadian Internet. Canadian Internet Registration Authority. Retrieved from: https://cira.ca/factbook/2014/the-canadian-internet.html

Chopik, W. J. (2016). The benefits of social technology use among older adults are mediated by reduced loneliness. Cyberpsychology, Behavior, and Social Networking, 19(9), 551-556.

Cotten, S. R., Ford, G., Ford, S., \& Hale, T. M. (2014). Internet use and depression among retired older adults in the United States: A longitudinal analysis. Journals of Gerontology Series B: Psychological Sciences and Social Sciences, 69(5), 763-771.

Davis, F. D. (1989). Perceived usefulness, perceived ease of use, and user acceptance of information technology. MIS quarterly, 319-340.

Elliot, A. J., Mooney, C. J., Douthit, K. Z., \& Lynch, M. F. (2013). Predictors of older adults' technology use and its relationship to depressive symptoms and well-being. Journals of Gerontology Series B: Psychological Sciences and Social Sciences, 69(5), 667-677.

Forsman, A. K., \& Nordmyr, J. (2017). Psychosocial links between Internet use and mental health in later life: a systematic review of quantitative and qualitative evidence. Journal of Applied Gerontology, 36(12), 1471-1518. 
Hargittai, E., Piper, A. M., \& Morris, M. R. (2018). From internet access to internet skills: digital inequality among older adults. Universal Access in the Information Society, $1-10$.

Helsper, E. J., \& Reisdorf, B. C. (2013). A quantitative examination of explanations for reasons for internet nonuse. Cyberpsychology, Behavior, and Social Networking, 16(2), 94-99.

Heo, J., Chun, S., Lee, S., Lee, K. H., \& Kim, J. (2015). Internet use and well-being in older adults. Cyberpsychology, Behavior, and Social Networking, 18(5), 268-272.

Hussain, A., Wenbi, R., da Silva, A. L., Nadher, M., \& Mudhish, M. (2015). Health and emergency-care platform for the elderly and disabled people in the Smart City. Journal of Systems and Software, 110, 253-263.

Jensen, J. D., King, A. J., Davis, L. A., \& Guntzviller, L. M. (2010). Utilization of internet technology by low-income adults: the role of health literacy, health numeracy, and computer assistance. Journal of aging and health, 22(6), 804-826.

Jung, Y., Peng, W., Moran, M., Jin, S.-A. A., McLaughlin, M., Cody, M., et al. (2010). Lowincome minority seniors' enrollment in a cybercafé: psychological barriers to crossing the digital divide. Educational gerontology, 36(3), 193-212

Keränen, N. S., Kangas, M., Immonen, M., Similä, H., Enwald, H., Korpelainen, R., \& Jämsä, T. (2017). Use of information and communication technologies among older people with and without frailty: a population-based survey. Journal of medical Internet research, 19(2).

Khosravi, P., Rezvani, A., \& Wiewiora, A. (2016). The impact of technology on older adults' social isolation. Computers in Human Behavior, 63, 594-603.

Laflamme, F., \& Karaganis, M. (2010, May). Implementation of responsive collection design for CATI surveys at Statistics Canada. In European Conference on Quality in Official Statistics (Q2010), Helsinki, May (pp. 4-6).

Lelkes, O. (2012). Happier and less isolated: internet use in old age. European Centre for Social Welfare Policy and Research.

Liang, T. H., Peng, J. L., \& Yu, C. Y. (2012). A simpler quality of e-life indicator: does the Internet have a positive impact on the quality of life in Taiwan. Quality \& Quantity, 46(4), 1025-1045.

Ma, Q., Chen, K., Chan, A. H. S., \& Teh, P. L. (2015, August). Acceptance of ICTs by older adults: a review of recent studies. In International Conference on Human Aspects of IT for the Aged Population (pp. 239-249). Springer, Cham.

Mazur, E., Signorella, M. L., \& Hough, M. (2018). The Internet Behavior of Older Adults. In Encyclopedia of Information Science and Technology, Fourth Edition (pp. 7026-7035). IGI Global.

McDonough, C. C. (2016). The effect of ageism on the digital divide among older adults. Journal of Gerontology and Geriatric Medicine, 2, 1-7.

Middleton, C. A., \& Sorensen, C. (2006). How connected are Canadians? Inequities in Canadian households' Internet access. Canadian Journal of Communication, 30(4).

Morris, A., Goodman, J., \& Brading, H. (2007). Internet use and non-use: views of older users. Universal access in the information society, 6(1), 43-57. 
Niehaves, B., \& Plattfaut, R. (2014). Internet adoption by the elderly: employing IS technology acceptance theories for understanding the age-related digital divide. European Journal of Information Systems, 23(6), 708-726.

Quan-Haase, A., Mo, G. Y., \& Wellman, B. (2017). Connected seniors: How older adults in East York exchange social support online and offline. Information, Communication \& Society, 20(7), 967-983.

Ramage-Morin, P. L. (2009). Medication use among senior Canadians. Health Reports, 20(1), 37

Ray, P. P. (2014, November). Home Health Hub Internet of Things (H 3 IoT): An architectural framework for monitoring health of elderly people. In Science Engineering and Management Research (ICSEMR), 2014 International Conference on (pp. 1-3). IEEE.

Statistics Canada. (2011). Table 2- Aboriginal Peoples in Canada: First Nations People, Metis and Inuit. 99-011-X2011001.

Statistics Canada (2017). Population Projections for Canada, Provinces and Territories. Occasional. 91-520-X. 2010001.

St-Pierre, M., \& Béland, Y. (2004, August). Mode effects in the Canadian Community Health Survey: A comparison of CAPI and CATI. In Proceedings of the Annual Meeting of the American Statistical Association, Survey Research Methods Section, August 2004.

Tsai, H. Y. S., Shillair, R., Cotten, S. R., Winstead, V., \& Yost, E. (2015). Getting grandma online: are tablets the answer for increasing digital inclusion for older adults in the US?. Educational gerontology, 41(10), 695-709.

van Boekel, L. C., Peek, S. T., \& Luijkx, K. G. (2017). Diversity in older adults' use of the internet: identifying subgroups through latent class analysis. Journal of medical Internet research, 19(5).

van Deursen, A. J., \& Helsper, E. J. (2015). A nuanced understanding of Internet use and nonuse among the elderly. European journal of communication, 30(2), 171-187.

Yu, R. P., Ellison, N. B., McCammon, R. J., \& Langa, K. M. (2016). Mapping the two levels of digital divide: Internet access and social network site adoption among older adults in the USA. Information, Communication \& Society, 19(10), 1445-1464.

Zickuhr, K., \& Madden, M. (2012). Older adults and internet use. Pew Internet \& American Life Project, 6 